\title{
Quantum-Implemented Selective Reconstruction of High-Resolution Images
}

\author{
Mitja Peruš \& Horst Bischof \\ Institute for Computer Vision \& Graphics, Graz University of Technology \\ Inffeldgasse 16 (2. OG), A-8010 Graz, Austria / \{perus,bischof\}@icg.tu-graz.ac.at \\ Loo Chu Kiong \\ Faculty of Engineering and Technology, Multimedia University (Melaka) \\ MY-75450 Melaka, Malaysia / ckloo@mmu.edu.my
}

\begin{abstract}
This paper proposes quantum image reconstruction. Input-triggered selection of an image among many stored ones, and its reconstruction if the input is occluded or noisy, has been simulated by a computer program implementable in a real quantum-physical system. It is based on the Hopfield associative net; the quantumwave implementation bases on holography. The main limitations of the classical Hopfield net are much reduced with the new, original - quantum-optical-implementation. Image resolution can be almost arbitrarily increased.
\end{abstract}

\section{Introduction}

There is growing evidence that quantum-physical systems could be harnessed for information processing, including specifically image recognition, in two ways:

- by Turing-machine-based quantum computing using quantum logic gates [1, 2, 3, 4,

- by quantum processing similar to those in (oscillatory) associative neural nets [5] (cf. [6, 7]).

This paper reports how it is possible to implement successful image recognition, as verified by our simulations, in a quantum holographic process 8 . Since the natural fundamental quantum-wave dynamics is harnessed, it allows much easier and cheaper physical realization with much bigger sizes and resolutions of images than the mainstream quantum-computing approaches [1, 2, 3, 4.

The main contribution of this paper is not to propose a generally-better image-recognition method, but to present its powerful alternative implementation into a quantum-wave medium (sec. 2), and to demonstrate its plausibility by computational experiments (sec. 3). Quantum-net's capacities of connectivity, parallelism, storage, associativity, speed and miniaturization are enormous, even much greater than in classical holography 9,10 .
In 11 it was shown how the Hopfield model with real-valued (thus not necessarily binary) activities of units / neurons, having linear (not sigmoid or signum) activation function, can be transformed into a quantum-holographic procedure [8] where the Hebbian memory-storage is replaced by multiple self-interferences of quantum plane-waves. This translation succeeded by the simplest variable exchange of the Hopfield's real-valued variables with the complex-valued variables changing according to sinusoids (waves) (cf. 6, 7]). Thereby, all input-to-output transformations are preserved. Thus, quantum-wave image recognition functions equivalently to Hopfield's one, only the implementation is much miniaturized enabling almost infinitely large neural-like networks.

Since the opposite translation, i.e. digitalization of holography, was done in the sixties of the $20^{t h}$ century to get the first computational associative memories, one might wonder what is new in the present proposal. The big experimental success of classical (optical, acoustic, microwave- etc., but also X-ray-, atom, electron-) holography 9 is widely known, but not also the recent fast development of quantum optics 12 which gave birth to quantum holography 8] (good "tutorial" in [13]). The latter promises to implement the well-known Hopfield model and its generalizations in a completely new framework where the former obstacles (memory-capacity limitations, problems with nonorthogonality of small-size inputs producing cross-talk) are very much reduced.

\section{Web of quantum waves}

Using neuro-quantum "isomorphisms", presented systematically in [16, and "numbers-to-waves" translation, as in 11, we transform the Hopfield-like associative neural net into quantum formalism (details in [11, 5]):

- Quantum wave-function $\Psi$ acts as net's state vector $\vec{q}$.

- Eigen-wave-functions $\psi^{k}(k=1, \ldots, P)$ act as Hopfield's pattern-bearing eigen-vectors (attractors) 
$\vec{v}^{k}$.

- The quantum Green-function propagator G replaces the Hebb memory matrix $\mathbf{J}$.

- Thus, sum of self-interferences $\psi^{k} \otimes \psi^{k}$ of quantum waves $\psi^{k}$ (that's the "hologram" G) implements the sum of auto-correlations of input-pattern configurations $\vec{v}^{k} \otimes \vec{v}^{k}$ (that's the content-addressable associative memory $\mathbf{J})$. ( $\otimes$ denotes tensor/outer product.)

The Hebb-equivalent expression for elements of $\mathbf{G}$ (i.e., the multiple cris-cross array $\sum_{k} \psi^{k} \otimes \psi^{k}$ implementing matrix $\mathbf{J}$ ) is:

$$
G_{h j}=\sum_{k=1}^{P} \psi_{h}^{k}\left(\psi_{j}^{k}\right)^{*}
$$

where $h$ and $j$ denote the unit / pixel / "neuron" / quantum point at locations $\vec{r}_{1}$ and $\vec{r}_{2}$ at time $t$ $(h, j=1, \ldots, N ; N$ can be almost infinite). The asterisk denotes complex conjugation (or, optically, phase conjugation).

After we have succeeded to encode patterns or images as eigen-states (attractors) $\psi^{k}$ into the quantum system prescribed by eq. (1), we can reconstruct one (say, $k_{0}^{t h}$ ) by presenting a new input similar to the $k_{0}^{t h}$ stored one:

$$
\begin{aligned}
\Psi_{h}^{\text {output }} & =\sum_{j=1}^{N} G_{h j} \Psi_{j}^{\text {input }}=\sum_{j=1}^{N}\left(\sum_{k=1}^{P} \psi_{h}^{k}\left(\psi_{j}^{k}\right)^{*}\right) \Psi_{j}^{\text {input }}= \\
& =\sum_{k=1}^{P}\left(\sum_{j=1}^{N}\left(\psi_{j}^{k}\right)^{*} \Psi_{j}^{\text {input }}\right) \psi_{h}^{k} \doteq \psi_{h}^{k_{0}}
\end{aligned}
$$

describes the resulting selective retrieval (recognition) of image $\vec{v}^{k_{0}}$ encoded in $\psi^{k_{0}}$. See detailed analysis in [11] or [5]. Eq. (2) is in analogy with [18. In the quantum Dirac notation, eq. (2) is, using $(\vec{a} \otimes \vec{b}) \vec{c}=$ $\langle\vec{b}, \vec{c}\rangle \vec{a}$ :

$$
\begin{aligned}
\left|\Psi^{\text {output }}\right\rangle & =\mathbf{G}\left|\Psi^{\text {input }}\right\rangle=\left(\sum_{k}\left|\psi^{k}\right\rangle\left\langle\psi^{k}\right|\right)\left|\Psi^{\text {input }}\right\rangle= \\
& =\sum_{k}\left\langle\psi^{k} \mid \Psi^{\text {input }}\right\rangle\left|\psi^{k}\right\rangle \doteq \psi^{k_{0}}
\end{aligned}
$$

We assume that we can encode images $\vec{v}^{k}$ into quantum plane waves (i.e., propagating sinusoidallychanging probability-distribution for measuring a photon ${ }^{1}$ at location $\vec{r}$ at time $t$ ):

$$
\psi^{k}(\vec{r}, t)=A^{k}(\vec{r}, t) e^{i \varphi^{k}(\vec{r}, t)}=A^{k} e^{\frac{i}{\hbar}\left(\vec{p}^{k} \vec{r}-E^{k} t\right)}
$$

We may choose the same constant amplitudes $A$, so that quantum phases (delays between wave-peaks) $\varphi$ encode the whole information. Let us take $A=1$ (or $A=1 / \sqrt{N}$ for convenient quantum normalization); so, $A_{j}^{k}=1$ (or another constant) for all $k, j$. (Various possibilities of amplitude and phase modulation

\footnotetext{
${ }^{1}$ The $k^{t h}$ mode of the photon has momentum $\vec{p}^{k}$ and energy $E^{k} ; \hbar$ is Planck's constant; $i=\sqrt{-1}$
}

see in [11.) The image-modulated laser-beam is thus: $\psi^{k}=\left(e^{i \varphi_{1}^{k}}, e^{i \varphi_{2}^{k}}, \ldots, e^{i \varphi_{N}^{k}}\right)$ where the number of wavefront points (wave peaks) is $N$.

The "isomorphism" of 11] allows us to exchange variables, $\vec{v}^{k} \leftrightarrow e^{i \varphi}$, giving $\psi_{j}^{k}=e^{i \varphi_{j}^{k}}$ instead of Hopfield-like $\psi_{j}^{k}=v_{j}^{k}$ (or $\psi_{j}^{k}=A_{j}^{k}$, respectively). With this exchange in equations (1) and (2), all the information-processing mathematics, verified by computer experiments of sec. 3 and [15, remains valid for sinusoid-encoded images also. Namely, because eq. (1) becomes

$$
G_{h j}=\sum_{k=1}^{P} e^{i \varphi_{h}^{k}} e^{-i \varphi_{j}^{k}}=\sum_{k=1}^{P} e^{i\left(\varphi_{h}^{k}-\varphi_{j}^{k}\right)}
$$

eq. (2) becomes

$$
\begin{aligned}
& e^{i \varphi_{h}^{\text {output }}}=\sum_{j=1}^{N}\left(\sum_{k=1}^{P} e^{i \varphi_{h}^{k}} e^{-i \varphi_{j}^{k}}\right) e^{i \varphi_{j}^{\text {input }}}= \\
= & \sum_{k=1}^{P}\left(\sum_{j=1}^{N} e^{-i \varphi_{j}^{k}} e^{i \varphi_{j}^{\text {input }}}\right) e^{i \varphi_{h}^{k}} \doteq e^{i \varphi_{h}^{k_{0}}}
\end{aligned}
$$

If images are almost orthogonal, a wave carrying an image (those among many stored ones which is the most similar to the newly input one) is selectively re= constructed.

There is a non-local information-exchange involved in this holographic process, which in our quantum case exploits the quantum interference web $(\mathbf{G})$ itself, not its static imprint onto a crystal plate as in classical holography 9 .

Our information-processing result can be extracted from $\psi^{k_{0}}$ using new quantum-optical (and computeraided) techniques for "ensemble"-measurement of observables or for quantum-holographic-(like) wavefront reconstruction [14]:

- quantum-phase estimation / engineering,

- wave-packet / wave-function reconstruction / sculpting / engineering,

- (coherent) quantum control / manipulation,

- quantum tomography.

\section{Computational experiments}

The purpose of these experiments is just to verify the theory on those basic aspects of the real quantum processing which can be simulated. Real quantumphysical systems provide performance (much) beyond what has been simulated by us up to now, and beyond what is simulable at all.

All experiments were done on a Pentium-4 1.3-GHz $\mathrm{PC}$ using the following algorithm programmed in MatLab with Image Processing Toolbox:

- $P$ images with index $k$ were encoded into $\vec{v}^{k}=$ $\left(\tilde{v}_{1}^{k}, \ldots, \tilde{v}_{N}^{k}\right)$ where a pixel's greyness is described by $\tilde{v}_{j}^{k} \in[0,255](j=1, \ldots, N)$. 

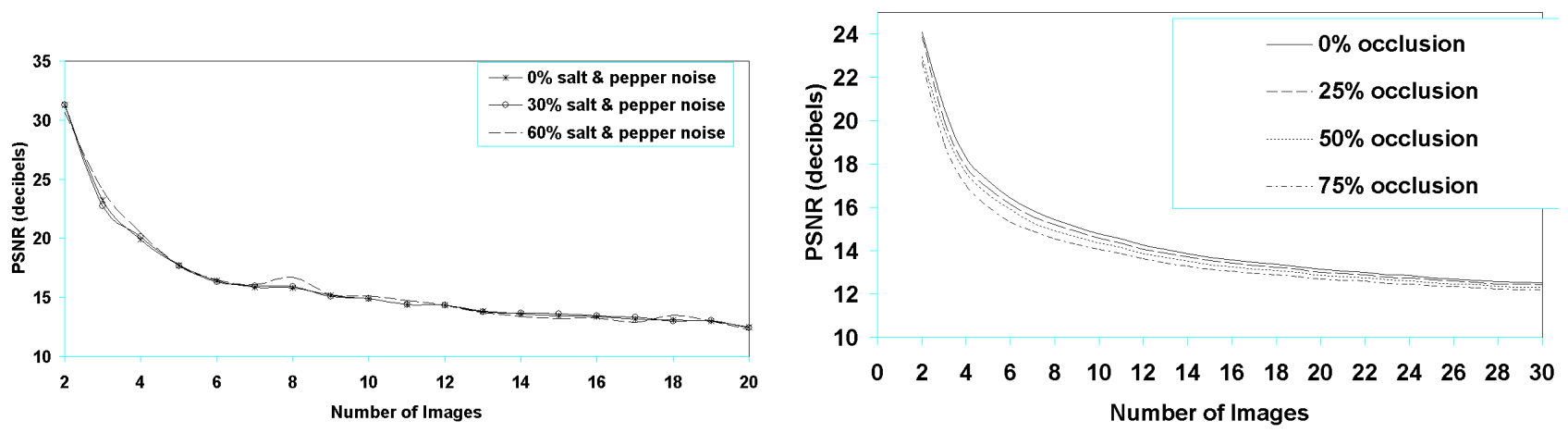

Figure 1: Plots of Peak Signal-to-Noise Ratio of reconstructed image from "query-image" versus number of simultaneously-stored images of (left) Chinese pictograms and (right) fingerprints, where (left) query is a Chinese pictogram with salt-and-pepper noise, and (right) query is an occluded fingerprint

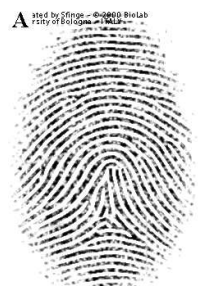

(a)

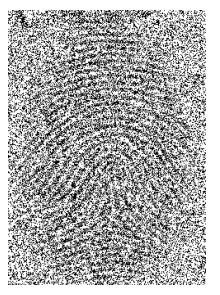

(b)

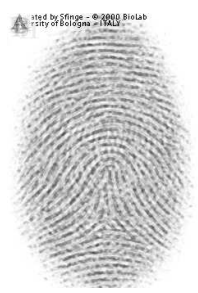

(c)

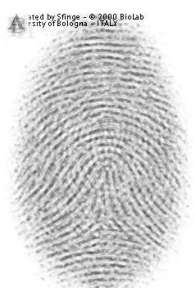

(d)

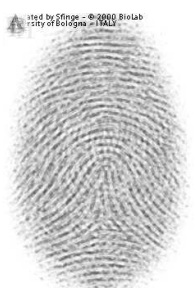

(e)

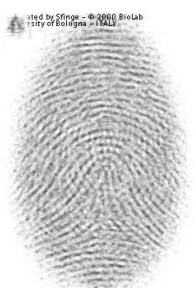

(f)

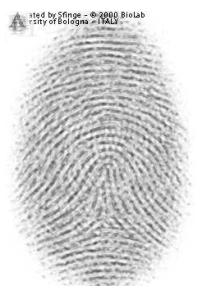

(g)

Figure 2: (a) An original image. (b) Original image (a) with $60 \%$ salt-and-pepper noise. (c)-(g) Image restored from memory of 10 different simultaneously-stored fingerprints after presentation of the "query-image" which is: (c) whole original image (a); (d) 25\%-occluded image (a); (e) 50\%-occluded (a); (f) 75\%-occluded (a); (g) noisy image (b).

- Images were preprocessed according to: $v_{j}^{k}=\tilde{v}_{j}^{k}-$ $\frac{1}{N} \sum_{j=1}^{N} \tilde{v}_{j}^{k}$ for each $k, j$. The resulting vector $\vec{v}^{k}$ was then normalized to satisfy $\sum_{j=1}^{N}\left(v_{j}^{k}\right)^{2}=1$. Such normalized $\vec{v}^{k}$ are assumed to be quantumimplemented into plane-wave/laser-beam $\psi^{k}$.

- Memory matrix, Eq. (1), was calculated ("storage stage").

- Later, in the "selective reconstruction stage", a new "query / recall-key" input was inserted. The network reacted as described in Eq. (3), or equivalently in Eq. (6). The "query-input" was completed (if partial initially) or corrected (if corrupted) based on memorized examples, and scaled back into $[0,255]$-range.

Quality of reconstructed image $\vec{v}$ was measured with Peak Signal-to-Noise Ratio (in dB; for 255 grey-values): $P S N R=20 \log _{10}\left(\frac{255}{R M S E}\right)$;

$R M S E=\sqrt{\frac{1}{N} \sum_{j=1}^{N}\left(v_{j}^{\text {original }}-v_{j}^{\text {reconstructed }}\right)^{2}}$.

We found that reconstruction-quality only slightly decreased with increasing number of images stored simultaneously, and that this behavior was similar regardless of the type of stored images and the type and rate of deviation of the query-image from the stored images. For two examples see Fig. 1 Compare these plots with Fig. [2 which demonstrates examples of "image recovery" from occlusion or noise. Indeed, the capability of selective reconstruction using memory is al- most the same for different rates of degradation (occlusion or corruption with noise) of the query-image or its deviation from the original stored image(s).

The performance is indeed holography-like - a small part of a hologram contains enough information about the whole pattern (stored in the hologram, our Eq. (5), in a parallel-distributed way) that the whole can be retrieved from the small part.

As evident from Fig. 2 the image which shared most pixels with the query-image, was selected from memory-matrix and reconstructed ("recognized"), being disrupted (merely) by cross-talk due to nonorthogonality of stored images. Such results, typical for associative nets and holography, were got also in the "mixed-set experiment" (Fig. 3). Here, 3 verydifferent sets of 10 different-content images, i.e. with big inter-set differences and small intra-set differences, were simultaneously stored. Cross-talk backgrounds can be seen in Figs. 3 (d-f), but the reconstructed images are not disrupted too much.

\section{Conclusions}

Our simulations confirm Hopfield-net's capabilities. The novelty of our simulations is reconsideration of Hopfield-net's characteristics in the age of powerful computers - early simulations of the eighties had a limited resolution of patterns rather than images. More- 


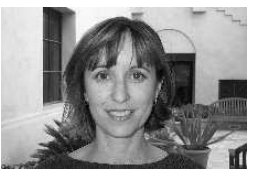

(a)

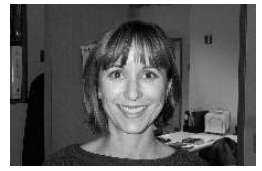

(b)

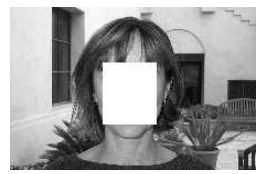

(c)

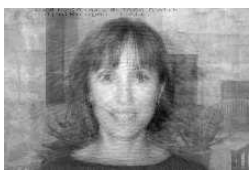

(d)

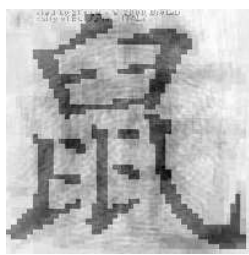

(e)

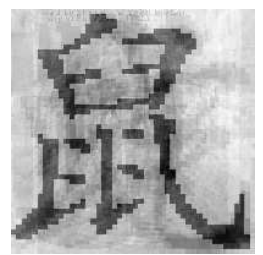

(f)

Figure 3: Reconstruction from 30 simultaneously-stored images (10 different Chinese pictograms and 10 different fingerprints as on Fig. 2(a) and 10 different face-poses like on (a)\&(b): "Query" (c) triggers reconstruction (d). (e)\&(f) Reconstructions from 25\%- and 50\%-occluded "query"-pictogram.

over, our original proposal of quantum-wave implementation opens a possibility of nets having up to almost infinite size, and of processing of huge / highresolution images. Therefore, Hopfield-net's cross-talk and storage limitations do not manifest (too) much for our practical needs. The first problem, cross-talk, is reduced since images with a huge number of pixels are usually almost orthogonal. The second problem, memory-capacity of the Hopfield model is limited (to $P \doteq 0.14 N)$, is much reduced with possibility of " astronomically big" $N$. Since databases include limited-size images, we have not yet been able to demonstrate the benefits of (quantum) huge-image processing, but they are evident even from classical holography [10, 9].

Instead of plane-waves, images could be encoded into Gabor wavelets 17] which are similar to quantum wave-packets. Other possible (great) improvements will be studied in the future.

Our proposal is enormously superior to other proposed quantum associative memories [2, 3, 1, 4, based on the mainstream of the quantum computing science using quantum-implemented logic gates, in the sense of simplicity, miniaturization, natural physical realizability of associative processing, memory capacity and dimensionality of data (specifically, size and resolution of images). Models 2, 3, 1, 4, are, however, more compatible with the mainstream attempts for an universal-purpose quantum computer, not merely for associative tasks which our model masters.

ACKNOWLEDGEMENTS: M.P. thanks for discussions to Prof. H.J. Caulfield, Prof. V. Bužek, Drs. C. Trugenberger and A. Vlasov. M.P. gratefully worked as EU Marie-Curie postdoc fellow (contract no. HPMF-CT-2002-01808).

\section{References}

[1] C. Trugenberger, "Quantum pattern recognition", Phys. Rev. Lett. 89 (2002) 277903. Phys. Rev. Lett. 87 (2001) 067901.

[2] D. Ventura and T. Martinez, "Quantum associative memory", Info. Sci. 124 (2000) 273-296.

[3] J. Howell, J. Yeazell and D. Ventura, "Optically simulating a quantum assoc. memory", Phys. Rev. A 62 (2000) 042303.

[4] R. Schützhold, "Pattern recognition on a quantum computer", xxx.lanl.gov/pdf/quant-ph/0208063 [cf., M. Sasaki et al., Phys. Rev. A 64 (2001) 022317].

[5] M. Peruš and S. Dey, "Quantum systems can implement content-addressable associative memory", Appl. Math. Lett. 13(8) (2000) 31-36.
[6] R. Spencer, "Bipolar spectral associative memories", IEEE Transac. Neural Netw. 12 (2001) 463-474.

[7] J. Sutherland, "Holographic model of memory, learning and expression", Int. J. Neural Sys. 1 (1990) 256-267.

[8] C. Leichtle et al., "Quantum state holography", Phys. Rev. Lett. 80 (1998) 1418-21. A. Abouraddy et al., "Quantum holography", Optics Express 9(10) (2001) 498-505. I. Averbukh et al., "Reconstructing wave-packets by quantumstate holography", Phys. Rev. A 59 (1999) 2163-73. [Cf.: P. Len et al.: J. Electron Spectrosc. 85 (1997) 145-158. N. Bhattacharya et al.: Phys. Rev. Lett. 88 (2002) 137901.]

[9] H. Bjelkhagen and H.J. Caulfield H.J. (eds.), Selected Papers on the Fundamental Techniques in Holography, SPIE Opt. Eng. Press, Bellingham (WA), 2001. R. Collier et al., Optical Holography, Academic Press, New York, 1971.

[10] F.T.S. Yu and S. Jutamulia (eds.), Optical Pattern Recognition, Cambridge Univ. Press, Cambridge, 1998.

[11] M. Peruš and H. Bischof, "Quantum-wave pattern recognition", in K. Chen et al. (eds.), Proceed. $7^{\text {th }}$ Joint Conf. Information Sciences 2003, Cary, NC, USA, pp. 1536-9; publ. by JCIS / Assoc. for Intelligent Machinery, Durham, 2003.

[12] W. Schleich, Quantum Optics in Phase Space, Wiley-VCH, Berlin, 2001.

[13] A. Granik and H.J. Caulfield, "Quantum holography", in: Holography, vol. IS 8 (Adv. Opt. Tech. Inst. Series), SPIE Opt. Eng. Press, Bellingham (WA), 1990, pp. 33-38.

[14] X. Chen and J. Yeazell, Phys. Rev. A 56 (1997) 2316-20. D. Goswami, Phys. Reports 376 (2003) 385-481. J.L. Krause et al., Phys. Rev. Lett. 79 (1997) 4978-81. D.T. Smithey et al., Phys. Rev. Lett. 70 (1993) 1244-7. B.C. Travaglione and G.J. Milburn, Phys. Rev. A 63 (2001) 032301.

[15] M. Peruš, "A computational study of reconstruction and anticipation capabilities of an associative neural net with large stored data-bases", Int. J. Computing Anticip. Sys. 13 (2002) 376-391.

[16] M. Peruš, "From neural to quantum associative nets", Neural Netw. World 10 (2000) 1001-13. [Nonlin. Phenom. in Complex Sys. 4 (2001) 157-193. Zeitschr. angew. Math. E6 Mech. 78, S 1 (1998) 23-26. Informatica 20 (1996) 173183.]

[17] T. Lee, "Image representation by 2D Gabor wavelets", IEEE Tr. Patt. Anal. \& Mach. Intell. 18(10) (1996) 1-13. R. Young, Wavelet Theory and Its Applications, Kluwer, Boston, 1993.

[18] V.M. Vapnik, Statistical Learning Theory, sec. 11.12.1: Reproducing kernel Hilbert spaces, J. Wiley, New York, 1998. 